\title{
Seasonal dependence in the solar neutrino flux
}

\author{
P. C. de Holanda \\ Instituto de Física Corpuscular - C.S.I.C., Departamento de Física Teòrica, Universitat of València, 46100 Burjassot, València, Spain \\ and Instituto de Física Gleb Wataghin, Universidade Estadual de Campinas, UNICAMP 13083-970 - Campinas, Brazil \\ C. Peña-Garay, M. C. Gonzalez-Garcia, and J. W. F. Valle \\ Instituto de Física Corpuscular - C.S.I.C., Departamento de Física Tèrica, Universitat of València, 46100 Burgassot, València, Spain
}

(Received 24 March 1999; published 8 October 1999)

\begin{abstract}
Mikheyev-Smirnov-Wolfenstein (MSW) solutions of the solar neutrino problem predict a seasonal dependence of the zenith angle distribution of the event rates, due to the nonzero latitude at the Super-Kamiokande site. We calculate this seasonal dependence and compare it with the expectations in the no-oscillation case as well as just-so scenario, in the light of the latest Super-Kamiokande 708-day data. The seasonal dependence can be sizable in the large mixing angle MSW solution and would be correlated with the day-night effect. This may be used to discriminate between MSW and just-so scenarios and should be taken into account in refined fits of the data. [S0556-2821(99)08419-2]
\end{abstract}

PACS number(s): 26.65. $+\mathrm{t}, 14.60 . \mathrm{Pq}$

The difference in the $\nu_{e}$ fluxes during the day and the night due to the regeneration of the $\nu_{e}$ in the Earth - the so-called day-night effect - is one of the milestones of the Mikheyev-Smirnov-Wolfenstein (MSW) solutions of the solar neutrino problem (SNP) [1,2]. This effect is negligible in the just-so picture [3]. Conversely, the oscillatory behavior of the conversion probability in the just-so scenario leads to seasonal-dependent event rates beyond the simple geometrical factor, due to variation of the Sun-Earth distance in different seasons of the year. Though recognized in the early days of the MSW effect $[2,4]$ this seasonal effect has been neglected in most discussions of the MSW solution to the SNP and has even been recently claimed to be absent in the MSW picture $[5,6]$.

Recent Super-Kamiokande data after 708 days [7] exhibit an excess of the number of events during the night [8]. Though not yet statistically significant this provides some hint in favor of the possible existence of a day-night effect. On the other hand there is also some hint for a seasonal variation in these data, especially for recoil electron energy above $11.5 \mathrm{MeV}$. While the former would be an indication in favor of the MSW solution, the latter would favor the just-so solution.

Here we call the attention to this interesting feature of the MSW solution, namely, that the expected MSW event rates do exhibit a seasonal effect due to the different night duration throughout the year at the experimental site, which leads to a seasonal-dependent $\nu_{e}$ regeneration effect in the Earth. Taking into account the relative position of the SuperKamiokande setup in each period of the year, we calculate the distribution of the events through the year both for the large mixing angle (LMA) and the small mixing angle (SMA) solutions to the SNP. We find that the effect can be as large as the one expected in the just-so scenario, especially in the LMA solution, where it amounts to $\sim 10 \%$ seasonal variation [see Eq. (12)] at the best fit point for the solar neutrino event rates given by Ref. [9]. For the SMA solution we find that the magnitude of the seasonal MSW effect is very small at the best fit point increasing as $\sin ^{2} 2 \theta$ increases within the $99 \%$ C.L. region. We illustrate this behavior in Figs. 1 and 2 and in Table I.

Let us now describe our calculation. For simplicity, let us consider the two-neutrino mixing case

$$
\nu_{e}=\cos \theta \nu_{1}+\sin \theta \nu_{2}, \nu_{\mu}=-\sin \theta \nu_{1}+\cos \theta \nu_{2} .
$$

We have determined the solar neutrino survival probability $P_{e e}$ in the usual way, assuming that the neutrino state arriving at the Earth is an incoherent mixture of the $\nu_{1}$ and $\nu_{2}$ mass eigenstates.

$$
P_{e e}=P_{e 1}^{\text {Sun }} P_{1 e}^{\text {Earth }}+P_{e 2}^{\text {Sun }} P_{2 e}^{\text {Earth }},
$$

where $P_{e 1}^{\text {Sun }}$ is the probability that a solar neutrino, that is created as $\nu_{e}$, leaves the Sun as a mass eigenstate $\nu_{1}$, and $P_{1 e}^{\text {Earth }}$ is the probability that a neutrino which enters the Earth as $\nu_{1}$ arrives at the detector as $\nu_{e}$. Similar definitions apply to $P_{e 2}^{\text {Sun }}$ and $P_{2 e}^{\text {Earth }}$.

The quantity $P_{e 1}^{\text {Sun }}$ is given, after discarding the oscillation terms, as

$$
P_{e 1}^{\mathrm{Sun}}=1-P_{e 2}^{\mathrm{Sun}}=\frac{1}{2}+\left(\frac{1}{2}-P_{\mathrm{LZ}}\right) \cos \left[2 \theta_{m}\left(r_{0}\right)\right],
$$

where $P_{\mathrm{LZ}}$ denotes the standard Landau-Zener probability $[10]$ and $\theta_{m}\left(r_{0}\right)$ is the mixing angle in matter at the neutrino production point. In our calculations of the expected event rates we have averaged this probability with respect to the production point assuming the production point distribution given in Ref. [11].

In order to obtain $P_{i e}^{\text {Earth }}$ we integrate the evolution equation in matter assuming a step-function profile of the Earth matter density. In the notation of Ref. [12], we obtain, for $P_{2 e}^{\text {Earth }}=1-P_{1 e}^{\text {Earth }}$

$$
P_{2 e}^{\text {Earth }}(\Phi)=(Z \sin \theta)^{2}+\left(W_{1} \cos \theta+W_{3} \sin \theta\right)^{2},
$$




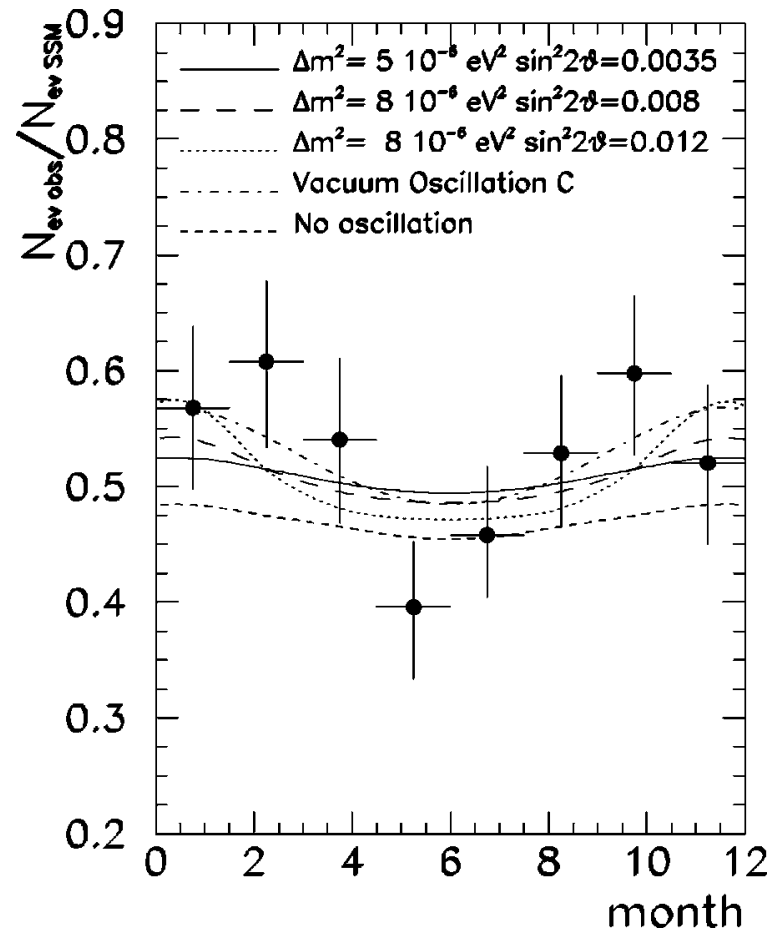

FIG. 1. Ratio of predicted event rate to the SSM prediction versus time of the year in Super-Kamiokande for various points in the SMA solution region of the SNP as labeled. We have normalized these three curves to the same yearly averaged event rate which corresponds to ${ }^{8} B$ flux normalization 0.7 for the best fit point in Ref. [9]. We also show the expectation in the absence of oscillations with ${ }^{8} B$ flux normalization of 0.47 (short dashed line) and the expected effect for vacuum oscillation solution $C$ in Ref. [6] (dash-dotted curve) together with the 708 Super-Kamiokande data points.

where $\theta$ is the mixing angle in vacuum and the Earth matter effect is included in the formulas for $Z, W_{1}$, and $W_{3}$, which can be found in Ref. [12]. $P_{2 e}^{\text {Earth }}$ depends on the amount of Earth matter traveled by the neutrino in its way to the detector, or, in other words, on its arrival direction which is usually parametrized in terms of the nadir angle, $\Phi$, of the sun at the detector site.

It is very important to realize that the daily range of variation of the nadir angle depends on the period of the year. As a result the quantity $P_{2 e}^{\text {Earth }}$ is seasonal dependent. This will, in turn, manifest itself as a seasonal dependence of the expected neutrino event rates. The general expression of the expected signal in the presence of oscillations at a given time $t, S^{\text {osc }}(t)$, is

$$
\begin{aligned}
S^{\mathrm{osc}}(t)= & \int d E_{\nu} \lambda\left(E_{\nu}\right)\left\{\sigma_{e}\left(E_{\nu}\right) P_{e e}\left(E_{\nu}, t\right)+\sigma_{x}\left(E_{\nu}\right)\right. \\
& \left.\times\left[1-P_{e e}\left(E_{\nu}, t\right)\right]\right\},
\end{aligned}
$$

where $E_{\nu}$ is the neutrino energy, $\lambda$ is the neutrino energy spectrum [13] with the latest normalization [14], $\sigma_{e}\left(\sigma_{x}\right)$ is the $\nu_{e}\left(\nu_{x}, x=\mu, \tau\right)$ interaction cross section in the standard model [15], and $P_{e e}$ is the $\nu_{e}$ survival probability, which varies in time through the interval of day and night along the

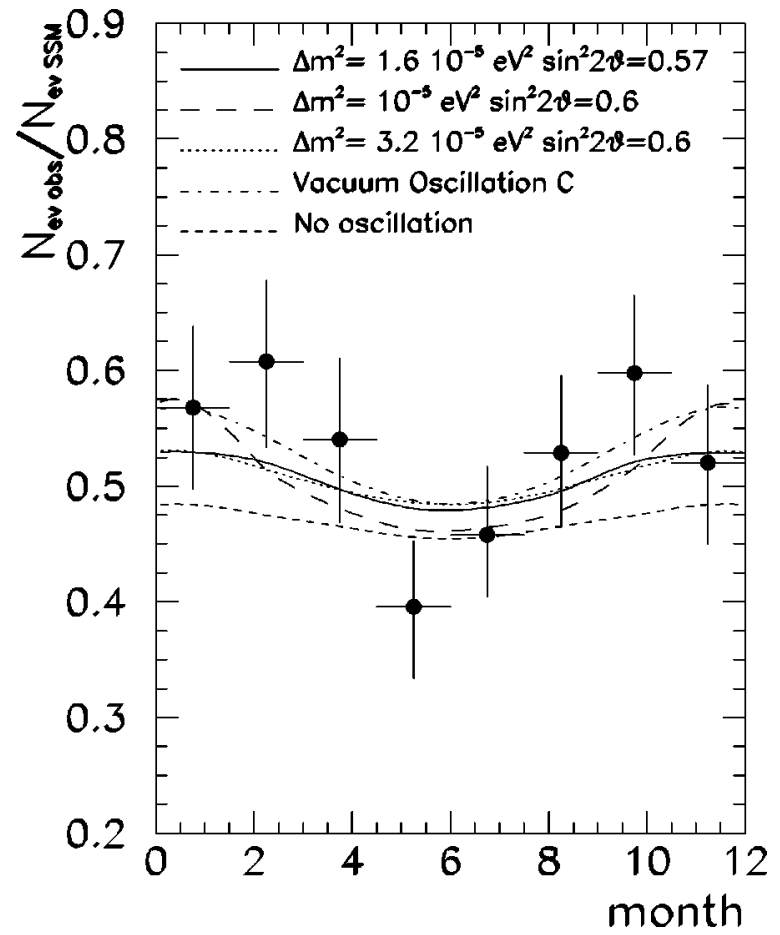

FIG. 2. Ratio of predicted event rate to the SSM prediction versus time of the year in Super-Kamiokande for various LMA solutions of the SNP labeled in the figure. These three curves are normalized to the same yearly averaged event rate corresponding to a ${ }^{8} B$ flux normalization 1.45 for the best fit point in Ref. [9]. We also show the expectation in the absence of oscillations with ${ }^{8} B$ flux normalization of 0.47 (short dashed line) and the expected effect for vacuum oscillation solution $\mathrm{C}$ in Ref. [6] (dash-dotted curve) together with the 708 Super-Kamiokande data points.

year. The expected signal in the absence of oscillations $S^{\text {no osc }}$ can be obtained from Eq. (5) by substituting $P_{e e}=1$.

The cross sections $\sigma_{e, x}$ are calculated including radiative corrections and must be corrected for energy threshold and resolution effects. In the calculation of the expected signal it is understood that the $\nu_{\alpha}-e$ cross sections $\sigma_{\alpha}(E)(\alpha=e, x)$ have to be properly corrected to take into account the detector energy resolution and the analysis window for each experiment. In Super-Kamiokande, the finite energy resolution implies that the measured kinetic energy $T$ of the scattered electron is distributed around the true kinetic energy $T^{\prime}$ according to a resolution function Res $\left(T, T^{\prime}\right)$ of the form [16]

$$
\operatorname{Res}\left(T, T^{\prime}\right)=\frac{1}{\sqrt{2 \pi} s} \exp \left[-\frac{\left(T-T^{\prime}\right)^{2}}{2 s^{2}}\right],
$$

where

$$
s=s_{0} \sqrt{T^{\prime} / \mathrm{MeV}},
$$

and $s_{0}=0.47 \mathrm{MeV}$ for Super-Kamiokande [7,17]. On the other hand, the distribution of the true kinetic energy $T^{\prime}$ for an interacting neutrino of energy $E_{\nu}$ is dictated by the differential cross section $d \sigma_{\alpha}\left(E_{\nu}, T^{\prime}\right) / d T^{\prime}$, that we take from Ref. [15]. The kinematic limits are 
TABLE I. Seasonal variation (in percent) of the ratio of predicted event rate in various oscillation scenarios to the SSM prediction.

\begin{tabular}{lccc}
\hline \hline Point & $\Delta m^{2}\left(\mathrm{eV}^{2}\right)$ & $\sin ^{2}(2 \theta)$ & Var $(\%)$ \\
\hline No-oscillation & & & 6 \\
MSW SMA & & & \\
\hline Best Fit Point & $5 \times 10^{-6}$ & $3.5 \times 10^{-3}$ & 6 \\
& $8 \times 10^{-6}$ & $8 \times 10^{-3}$ & 10 \\
& $8 \times 10^{-6}$ & $1.2 \times 10^{-2}$ & 20 \\
MSW LMA & & & \\
\hline Best Fit Point & $1.6 \times 10^{-5}$ & 0.57 & 10 \\
& $1 . \times 10^{-5}$ & 0.6 & 22 \\
Vacuum Solutions & $3.2 \times 10^{-5}$ & 0.6 & 9 \\
\hline C & & & 15 \\
D & $4.4 \times 10^{-10}$ & 0.93 & 12 \\
A & $6.4 \times 10^{-10}$ & 1 & 9 \\
\hline \hline
\end{tabular}

$$
0 \leqslant T^{\prime} \leqslant \bar{T}^{\prime}\left(E_{\nu}\right), \quad \bar{T}^{\prime}\left(E_{\nu}\right)=\frac{E_{\nu}}{1+m_{e} / 2 E_{\nu}} .
$$

For assigned values of $s_{0}, T_{\min }$, and $T_{\max }$, the corrected cross section $\sigma_{\alpha}\left(E_{\nu}\right)$ is defined as

$$
\sigma_{\alpha}\left(E_{\nu}\right)=\int_{T_{\min }}^{T_{\max }} d T \int_{0}^{\bar{T}^{\prime}\left(E_{\nu}\right)} d T^{\prime} \operatorname{Res}\left(T, T^{\prime}\right) \frac{d \sigma_{\alpha}\left(E_{\nu}, T^{\prime}\right)}{d T^{\prime}} .
$$

Finally, in order to compare our results with the recent data from the Super-Kamiokande Collaboration, we must also include the geometrical seasonal neutrino flux variation due to the variation of the Sun-Earth distance $(L \approx 1.5$ $\left.\times 10^{13} \mathrm{~cm}\right)$ arising from the Earth's orbit eccentricity because the neutrino fluxes in Eq. (5) are yearly averages. In order to account for this effect we assume a $1 / L^{2}$ dependence of the flux. Notice that the Super-Kamiokande data are presented as ratio of observed events over the expected number in the standard solar model where this expected number of events does not include the geometrical variation. Thus we must compare the experimental points with the predictions

$$
\frac{N_{\mathrm{osc}}\left(t_{0}, \Delta t\right)}{N_{\mathrm{no} \mathrm{osc}}(\Delta t)}=\frac{\int_{t_{0}-\Delta t / 2}^{t_{0}+\Delta t / 2} d t\left[S^{\mathrm{osc}}(t) / \hat{L}^{2}(t)\right]}{\Delta t S^{\mathrm{no} \mathrm{osc}}}
$$

where

$$
\hat{L}(t)=\left[1-\epsilon \cos 2 \pi \frac{t}{T}\right]
$$

and $\epsilon=0.0167$ is the eccentricity of Earth's orbit around the Sun, and $T=1$ year.

We now turn to our results. In order to study the behavior of the seasonal variation we have explored the parameter space around the small and large mixing angle solutions, SMA and LMA, respectively. We find that depending on the values of the mass and mixing angle, one may get a sizeable enhancement of the geometrical effect.

In Fig. 1 we present the expected event numbers in the recoil electron energy range $T_{\min }=11.5 \mathrm{MeV}$ up to $T_{\max }$ $=20 \mathrm{MeV}$, plotted versus the period of the year for different points in the SMA solution region of the SNP divided by the Bahcall-Basu-Pinsonneault 1998 (BBP98) standard solar model (SSM) predictions in the absence of neutrino conversions [14]. We plot the expected behavior for three points: the best fit point obtained by [9] with an arbitrary ${ }^{8} B$ flux, $\Delta m^{2}=5 . \times 10^{-6} \mathrm{eV}^{2}$ and $\sin ^{2} 2 \theta=3.5 \times 10^{-3}$, a point inside the $99 \%$ confidence level allowed region with $\Delta m^{2}=8$ $\times 10^{-6} \mathrm{eV}^{2}$ and $\sin ^{2} 2 \theta=8 \times 10^{-3}$ and a near point with $\Delta m^{2}=8 \times 10^{-6} \mathrm{eV}^{2}$ and $\sin ^{2} 2 \theta=1.2 \times 10^{-2}$. We have normalized these three curves to the same yearly averaged event rate. This corresponds to ${ }^{8} B$ flux normalization 0.7 for the best fit point as obtained from the global fit with free ${ }^{8} B$ flux in Ref. [9]. For the sake of comparison we also plot the expected behavior in the absence of oscillations with ${ }^{8} B$ flux normalization of 0.47 as well as the best fit point for the vacuum solution $\mathrm{C}$ of Ref. [6]. As seen in the figure the seasonal effect is comparable to the expectation in the absence of oscillation at the best fit point of the SMA solution and it increases as the mixing angle increases. In Table I we show the seasonal variation (in percent) defined as

$$
\operatorname{Var} \equiv 2 \frac{R_{\max }-R_{\min }}{R_{\max }+R_{\min }}
$$

for the different MSW and vacuum solutions of the SNP where $R(t)=N_{\text {osc }}(t) / N_{\mathrm{SSM}}$. We find that for the SMA solution the effect increases as one increases $\sin ^{2} 2 \theta$. For example for $\sin ^{2} 2 \theta=0.008$, still within the $99 \%$ C.L. allowed region, it reaches $10 \%$ and for $\sin ^{2} 2 \theta=0.012$ it gets to be as large as $20 \%$. Of course, since the seasonal effect is induced by the variation of the regeneration in the Earth along the year, the effect is large only in the parameter region where the daynight effect is not negligible, which corresponds to larger mixing angle values [18]. Note that in the SMA region the points we have chosen in order to illustrate the possible seasonal variation in the MSW picture are consistent with the measured yearly average day-night asymmetry.

Now we turn to the LMA solution of the SNP where the effects are potentially larger. Our results for this case are displayed in Fig. 2. Again, we plot the expected behavior for three characteristic points: the best fit point obtained by [9] with an arbitrary ${ }^{8} B$ flux $\left(\sin ^{2} 2 \theta=0.57, \Delta m^{2}=1.6\right.$ $\times 10^{-5} \mathrm{eV}^{2}$ ), a point inside the $99 \%$ confidence level allowed region with $\Delta m^{2}=1 \times 10^{-5} \mathrm{eV}^{2}$ and $\sin ^{2} 2 \theta=0.6$ and a point inside the allowed region where the expected average day-night asymmetry is smaller, $\Delta m^{2}=3.2 \times 10^{-5} \mathrm{eV}^{2}$ and $\sin ^{2} 2 \theta=0.6$. We have normalized these three curves to the same yearly averaged event rate. This corresponds to a ${ }^{8} \mathrm{~B}$ flux normalization 1.45 for the best fit point as obtained from the global fit with free ${ }^{8} B$ flux in Ref. [9]. We also plot the expected behavior in the absence of oscillations with ${ }^{8} B$ flux normalization of 0.47 and the best fit vacuum solution $\mathrm{C}$ 
from Ref. [6]. In Table I we show the variation (in percent) corresponding to these points. As seen in the table the effect at the best fit point of the LMA solution (10\%) is comparable with the corresponding effect in some of the favored vacuum oscillation solutions. In the LMA solution region the seasonal variation is very mildly dependent on the mixing angle while presents an oscillatory variation with $\Delta m^{2}$. We must bear in mind, however, that in the lower $\Delta m^{2}$ part of the LMA solution region, the expected yearly average day-night asymmetry is in conflict with the existing data [18]. Finally let us comment on the effect of an enhanced hep neutrino flux as suggested in Ref. [19] in order to account for the recent Super-Kamiokande measurements of the energy spectrum. We find that even with large hep enhancement factors of 20 or more, the expected modifications of our results near the best fit points both for the SMA and LMA are small.

To summarize, we have shown that MSW solutions of the solar neutrino problem can lead to sizeable seasonal depen- dence of the event rates at the Super-Kamiokande detector in the large mixing angle region and this should be taken into account in refined fits of the data where the day-night analysis is also performed. The MSW seasonal effect is correlated with the day-night asymmetry [18] and may potentially be useful in order to pinpoint the underlying mechanism involved in the explanation of the solar neutrino anomaly, discriminating between different solutions. For example, the non-observation of the day-night effect and the confirmation of seasonal-dependent rates would provide an indication for the just-so picture. Conversely, a possible confirmation of a seasonal dependence accompanied by the day-night effect would point towards a LMA MSW-type solution.

We are grateful to E. Akhmedov and C. Yanagisawa for useful comments. This work was supported by Spanish DGICYT under Grant No. PB95-1077, by the European Union TMR network ERBFMRXCT960090. P. C. de Holanda was supported by FAPESP and PRONEX (Brazil).
[1] S. P. Mikheyev and A. Yu. Smirnov, in '86 Massive Neutrinos in Astrophysics and in Particle Physics, Proceedings of the Sixth Moriond Workshop, edited by O. Fackler and J. Trân Thanh Vân (Editions Frontières, Gif-sur-Yvette, 1986), p. 355.

[2] S.P. Mikheyev and A.Yu. Smirnov, Usp. Fiz. Nauk 153, 3 (1987) [Sov. Phys. Usp. 30, 759 (1987)]; J. Bouchez et al., Z. Phys. C 32, 499 (1986); A. Dar et al., Phys. Rev. D 35, 3607 (1987); E.D. Carlson, ibid. 34, 1454 (1986); A.J. Baltz and J. Weneser, ibid. 50, 5971 (1994); A.J. Baltz and J. Weneser, ibid. 51, 3960 (1994); P.I. Krastev, hep-ph/9610339; M. Maris and S.T. Petcov, Phys. Rev. D 56, 7444 (1997); J.N. Bahcall and P.I. Krastev, Phys. Rev. C 56, 2839 (1997).

[3] S.L. Glashow and L.M. Krauss, Phys. Lett. 19B, 199 (1987); S. Pakvasa and J. Pantaleone, Phys. Rev. Lett. 65, 2479 (1990); A. Acker, S. Pakvasa, and J. Pantaleone, Phys. Rev. D 43, 1754 (1991); V. Barger et al., ibid. 43, 1110 (1991); V. Barger et al., Phys. Rev. Lett. 69, 3135 (1992); P.I. Krastev and S.T. Petcov, Phys. Lett. B 299, 99 (1993); S.L. Glashow, P.J. Kernan, and L. Krauss, ibid. 445, 412 (1999).

[4] A.J. Baltz and J. Weneser, Phys. Rev. D 35, 528 (1987); A.J. Baltz and J. Weneser, ibid. 37, 3364 (1988).

[5] M. Maris and S.T. Petcov, Phys. Lett. B 457, 319 (1999).

[6] V. Barger and K. Whisnant, Phys. Lett. B 456, 54 (1999).

[7] Super-Kamiokande Collaboration, Y. Fukuda et al., Phys. Rev. Lett. 82, 1810 (1999).

[8] Y. Suzuki, talk given at 17th International Workshop on Weak Interactions and Neutrinos, Cape Town, South Africa, January
1999; M.B. Smy, talk at DPF-99, Los Angeles, California, 1999, hep-ex/9903034.

[9] J.N. Bahcall, P.I. Krastev, and A.Yu. Smirnov, Phys. Rev. D 58, 096016 (1998).

[10] S.T. Petcov, Phys. Lett. B 200, 373 (1988); P.I. Krastev and S.T. Petcov, ibid. 207, 64 (1988).

[11] URL http://www.sns.ias.edu/jnb/SNdata/Export/Models /bp98flux.dat; J.N. Bahcall, S. Basu, and M.H. Pinsonneault, Phys. Lett. B 433, 1 (1998).

[12] E.Kh. Akhmedov, Nucl. Phys. B538, 25 (1999).

[13] J.N. Bahcall, E. Lisi, D.E. Alburger, L. De Braeckeleer, S.J. Freedman, and J. Napolitano, Phys. Rev. C 54, 411 (1996).

[14] J.N. Bahcall, S. Basu, and M.H. Pinsonneault, Phys. Lett. B 433, 1 (1998).

[15] J.N. Bahcall, M. Kamionkowsky, and A. Sirlin, Phys. Rev. D 51, 6146 (1995).

[16] See, e.g., J.N. Bahcall, P.I. Krastev, and E. Lisi, Phys. Rev. C 55, 494 (1997).

[17] B. Faid, G.L. Fogli, E. Lisi, and D. Montanino, Astropart. Phys. 10, 93 (1999).

[18] M.C. Gonzalez-Garcia, P.C. de Holanda, C. Peña-Garay, and J.W.F. Valle, hep-ph/9906469; M. C. Gonzalez-Garcia and A. Yu. Smirnov, Proceedings of International Workshop on Particles in Astrophysics and Cosmology: Theory to Observation Valencia, 1999, Nucl. Phys. B (Proc. Suppl.) (to be published), edited by V. Berezinsky, G. Raffelt, and J. W. F. Valle.

[19] J.N. Bahcall and P.I. Krastev, Phys. Lett. B 436, 243 (1998); R. Escribano et al., ibid. 444, 397 (1998). 